\title{
EXPERIENCIA EXITOSA EN LA IMPLEMENTACIÓN DEL SISTEMA GESTIÓN DE LA INOCUIDAD ALIMENTARIA ISO 22000:2005
}

\section{Engel Román Morales Ruiz}

Licenciado en Química

Supervisor de inocuidad alimentaria, Ingenio Monte Rosa

emorales.ylrpo@pantaleon.com

\section{RESUMEN}

El ingenio Monte Rosa S.A (MSA), cumpliendo con su futuro visionario de llegar a ser una de las 10 empresas más importantes en el mercado de edulcorantes en el mundo para el año 2030, decidió en el año 2007 certificarse bajo la norma ISO 22000:2005. Esto para asegurar la salud de los consumidores de los productos elaborados por la empresa. La norma ISO 22000 establece los requerimientos que debe cumplir un sistema de gestión de la inocuidad alimentaria (SGIA) en una organización dentro de la cadena alimentaria. El sistema ISO 22000 de MSA está basado en un robusto sistema de gestión de la calidad ISO 9001:2000 desde el año 2004, certificado por Global-QMI. Además, cuenta con un sistema de Análisis de Riesgos y Puntos críticos de control (HACCP), implementado en la empresa desde el año 2005, el cual está compuesto por los prerrequisitos: Buenas Prácticas Agrícolas (BPA) y Buenas Prácticas de Manufactura (BPM). El sistema de gestión de inocuidad alimentaria permite demostrar el cumplimiento de los requisitos de gestión eficaz de los procesos, la implementación de un sistema HACCP; así como el control de los procesos de producción y de especificaciones de producto. La implementación de este sistema ha ayudado a Monte Rosa S.A a mantener niveles altos de inocuidad alimentaria, atrayendo nuevos clientes y sirviendo como benchmarking a ingenios centroamericanos, identificando oportunidades de mejora y cumpliendo con las normativas y leyes del país.

\section{INTRODUCCIÓN}

Monte Rosa S.A es un ingenio azucarero que forma parte del grupo Pantaleón, el cual apunta a cumplir con sus lineamientos estratégicos de rentabilidad, desarrollo y diversificación geográfica.

Por tanto, en el año 2005 decidió buscar la certificación en la ISO 22000:2005, la cual es una norma para desarrollar e implementar sistemas de gestión de la inocuidad alimentaria, cuya intención final es conseguir una armonización internacional entre las muchas normas existentes y ser una herramienta para lograr la mejora continua en la inocuidad alimentaria a lo largo de la cadena de suministros de los productos alimenticios.

El cumplimiento de los requisitos de esta norma aseguran el cumplimiento de los requisitos de gestión eficaz de los procesos, la implementación de un sistema HACCP; así como el control de los procesos de producción y de especificaciones de producto.

\section{DESARROLLO}

El proceso de certificación en ISO 22000:2005 dio inicio en septiembre del 2005 con la publicación de la norma. Se realizaron todas las actividades necesarias de implementación y en marzo del 2006 el ingenio se sometió a un proceso riguroso de auditoria externa, con una semana de duración.

Esta etapa fue ejecutada por la firma auditora QMI, la cual nos avaló y certificó. Cabe mencionar que no es ISO directamente quien nos otorga un certificado, ya que ISO es la organización internacional de normalización creadora de las normas y QMI es el ente certificador acreditado para certificar sistemas de gestión basados en las normas ISO. 
El departamento de gestión de calidad e inocuidad se encarga de dar seguimiento al cumplimiento y mantenimiento de los sistemas de gestión, así como investigar sobre nuevos métodos y sistemas que le permitan a la empresa seguir siendo competitiva en el mercado actual.

\section{Evidencias de compromiso gerencial y liderazgo}

Para que un Sistema de Gestión se implemente y se mantenga con éxito, es preciso que exista un compromiso gerencial con su aplicación, en ausencia de éste no es posible alcanzar resultados.

Desde el inicio de la implementación del Sistema de Gestión de Calidad en Monte Rosa en el año 2004, el grupo de gerentes se involucró decididamente en el proyecto, lo cual fue clave para poner en marcha el sistema.

Esta fórmula de compromiso y liderazgo le ha permitido a Monte Rosa seguir implementando nuevos Sistemas de Gestión, tal es el caso del Sistema de Gestión de Inocuidad Alimentaria (SGIA) basado en la norma ISO 22000:2005.

También la motivación en todos los niveles fue necesaria; para este tipo de proyectos todos los jefes del ingenio se involucraron y su aporte fue indispensable para hacer generar compromiso y participación en el resto de trabajadores.

Para el Comité de Gerentes de Monte Rosa es muy importante participar directamente en la gestión del sistema, evidencia de esto son las reuniones semanales de los gerentes donde se tratan los temas relacionados a los Sistemas de Gestión implementados, así como el seguimiento trimestral de objetivos e indicadores de procesos.
Para la implementación de esta norma intervinieron todos lo trabajadores de la empresa cortadores de caña, operarios de fábrica, mecánicos, personal de las labores agrícolas, supervisores, coordinadores, jefes de proceso y gerentes de área, para quienes mantener esta certificación significa el compromiso de inocuidad desde el campo hasta el consumidor final.

En la figura 1 se muestra la integración de prerrequisitos en la cadena productiva del azúcar.

Por otro lado, se involucró a los proveedores de productos y servicios, a los cuales se les envió por escrito el compromiso de la empresa con la inocuidad y los requerimientos que debían de cumplir sus suministros.

Se aplicó un plan de comunicación que requirió la creación de un departamento de comunicación interna encargado de elaborar los mensajes y proponer estrategias de comunicación a todos los involucrados.

\section{En busca de oportunidades de mejora}

Las auditorías internas en MSA son una herramienta de autocontrol, tanto del sistema de gestión de calidad como del sistema de gestión de inocuidad, para asegurar que la organización HACE lo que DICE que HACE.

Antes de cada auditoría, los ejecutores se preparan revisando los procedimientos, instructivos y demás documentos correspondientes al proceso, donde se han plasmado los requisitos de la organización y de las normas tanto ISO 9001:2000 como ISO 22000:2005.

Figura 1. A. Buenas prácticas en campo. B. Sanidad en instalaciones. $C$. Higiene del personal.

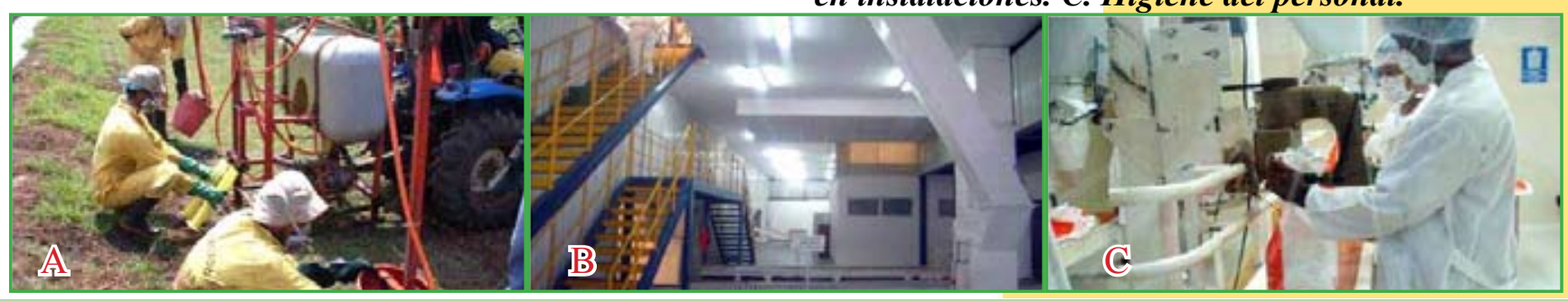


Durante la realización de la auditoría, por medio de entrevistas al personal, se verifica el cumplimiento de los procedimientos. También se revisan a detalle los registros de los procesos, ya que estos evidencian la ejecución de lo descrito en los procedimientos.

Es muy importante señalar, que el principal objetivo de las auditorías internas es encontrar oportunidades de mejora en los procesos y por esta razón, al momento de realizar la auditoría, todo el personal de Monte Rosa S.A. es conciente de que se debe mantener un espíritu de colaboración con el auditor.

El equipo de auditores de Monte Rosa S.A. es un equipo multidisciplinario conformado por 60 miembros, los cuales pertenecen a las diferentes áreas de la empresa y su selección ha sido una decisión de la Alta Dirección, para la cual se han tomado en cuenta entre otros los siguientes criterios en los candidatos seleccionados:

- Comunicación

- Análisis

- Objetividad

- Amabilidad

- Tenacidad

Los auditores internos año con año reciben entrenamiento de actualización en técnicas de auditoría, así como en aspectos de las normas implementadas.

\section{Tecnología de punta}

La planificación de productos inocuos y la búsqueda de la mejora continua de los sistemas han llevado a Monte Rosa S.A a buscar tecnología de punta que le permita ser eficaz con sus indicadores de calidad e inocuidad de los productos.
Como ejemplo de la tecnología utilizada para garantizar la eliminación de los peligros a la inocuidad se pueden mencionar los detectores de metales (A), rejillas magnéticas (B) y filtros de banda (C), mostrados en la figura 2.

Estos recursos tecnológicos se apoyan con personal capacitado y comprometido, pues la fusión de ambos es lo que garantiza que la política de calidad se implemente.

\section{CONCLUSIONES}

La implantación de un sistema de inocuidad alimentaría involucra el compromiso de la alta dirección, quienes a través de su liderazgo crean una política de inocuidad, la cual es comunicada a todo el personal de la empresa.

En el Ingenio Monte Rosa, somos responsables con la producción de alimentos seguros para nuestros consumidores, esto lo garantizamos con la certificación ISO 22000:2005, basada en Sistema de Análisis de riesgos y Puntos críticos de control.

Somos una empresa para enfrentar nuevos retos que satisfagan las expectativas de nuestros clientes.

\section{Figura 2. A) Detector de metales B) Rejilla metálica C) Filtros de banda}

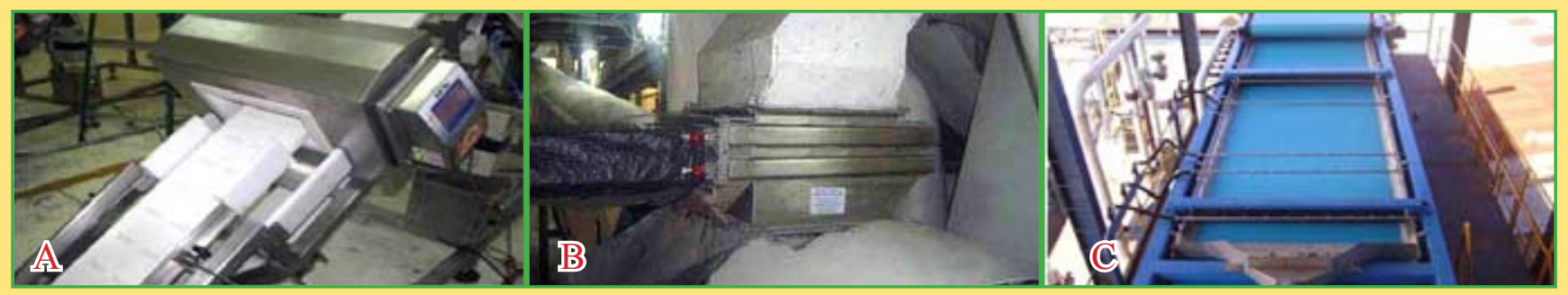

